DOI: https://doi.org/10.32839/2304-5809/2021-5-93-34

УДК 519.6

Мотайло А.П.

Херсонська державна морська академія

\title{
КУБАТУРНА ФОРМУЛА НА ОКТАЕДРІ
}

\begin{abstract}
Анотація. При розв'язанні граничних задач математичної фрізики методом скінченних елементів у тривимірній області з використанням решіток тетраедрально-октаедральної структури існуе необхідність отримання формул чисельного інтегрування по області октаедра. У даній роботі побудовано кубатурну формулу на октаедрі, яка є точною для алгебраїчних тривимірних поліномів третього, п'ятого та сьомого степенів. При цьму точність отриманої фрормули визначається вибором відповідних груп вузлів інтерполяції, які розташовані на осях симетрії даного багатогранника. Додавання певної групи вузлів приводить до збільшення степеня алгебраїчної точності від третього до сьомого. Визначено оптимальні параметри отриманої формули за кількістю вузлів інтерполяції, додатними ваговими коефіцієнтами та наявністю вузлів за межами області інтегрування при різних значеннях степеня тривимірного алгебраїчного полінома. Отримано оцінку залишкового члена кубатурної формули для підінтегральних функцій, які належать класу неперервно-диференційованих функщій до порядку 4, 6, 8 відповідно в області октаедра. Дана фрормула може бути використана для розрахунку скінченно-елементних матриць дискретної моделі задачі, забезпечує високий порядок точності обчислень та є ефективною за часовою складністю алгоритму методу скінченних елементів.
\end{abstract}

Ключові слова: октаедр, базисні функції, метод скінченних елементів, алгебраїчні поліноми.

Motailo Anzhelika

Kherson State Maritime Academy

\section{CUBATURE FORMULA ON THE OCTAHEDRON}

Summary. When solving boundary value problems of mathematical physics by the finite element method in the three-dimensional domain using lattices of tetrahedral-octahedral structure, there is a need to obtain formulas for numerical integration in the field of octahedron. The question of determining the elements of matrices that are related to the quadratic functional is important when writing computational programs of the finite element method. Therefore, the software that implements this method is based on accurate and efficient numerical methods of integration in the finite element, as well as aimed at minimizing the number of operations and optimal use of RAM and external computer memory. In this paper, we construct a cubature formula on an octahedron that is exact for algebraic three-dimensional polynomials of the third, fifth, and seventh degrees. The accuracy of the obtained formula is determined by the choice of appropriate groups of interpolation nodes, which are located on the axes of symmetry of this polyhedron. All nodes are divided into four groups: interpolation nodes, which lie on the axes of the octahedron, passing through opposite vertices of the polyhedron, located at a given distance from its center; nodes that are the points of intersection of a sphere of radius $q$ with the axes of the octahedron, passing through the middle of the opposite edges of the polyhedron; nodes located at the points of intersection of a sphere of radius $r$ with the axes of the octahedron, passing through the centers of gravity of opposite faces of the polyhedron; the center of the octahedron, located at the beginning of the local coordinate system. Adding a certain group of nodes leads to an increase in the degree of algebraic accuracy from the third to the seventh. The optimal parameters of the obtained formula are determined by the number of interpolation nodes, positive weights and the presence of nodes outside the integration domain at different values of the degree of three-dimensional algebraic polynomial. The resulting formula satisfies the condition of positive weights, and is the minimum number of interpolation nodes for algebraic polynomials of third degree and has two different sets of coordinates of nodes and weights for algebraic polynomials of fifth and seventh degrees. For polynomials of the fifth degree it is possible to choose a formula, all nodes of which belong to the integration domain and correspond to the values of parameters $p_{2}, r_{2}$. For an algebraic polynomial of the seventh degree, regardless of the choice of parameters of the cubature formula, one of the groups of nodes does not belong to the domain of integration. An estimate of the residual term of the cubature formula for subintegral functions belonging to the class of continuously differentiated functions to the order of 4,6 , 8 , respectively, in the octahedron domain is obtained. This formula can be used to calculate finite-element matrices of a discrete model of the problem, provides a high order of accuracy of calculations and is time-efficient algorithm of the finite element method.

Keywords: octahedron, basic functions, finite element method, algebraic polynomials.

Постановка проблеми. Сьогодні чисельне інтегрування стає однією 3 важливих частин методу скінченних елементів (MCE). Питання визначення елементів матриць, які пов'язані з квадратичним функціоналом, $е$ важливим при написанні обчислювальних програм MCE. Тому програмне забезпечення, яке реалізуе MCE, базуеться на точних та ефективних чисельних методах інтегрування по області скінченного елемента, а також направлене на мінімізацію числа операцій та оптимальне використання оперативної та зовнішньої пам'яті обчислювальної техніки.

Відомо, що у випадку, коли задача є тривимірною, при розрахунку дискретної моделі значно зростає часова складність алгоритму МСE. При цьому одним із способів оптимізації скінченно-елементних розрахунків є використання альтернативних решіток при дискретизації області розв’язуваної граничної задачі. 
Аналіз останніх досліджень і публікацій. У роботах [1-3] для створення дискретних моделей у $3 \mathrm{D}$ застосовано тетраедрально-октаедральні решітки. При цьому у роботі [3] досліджено умови збіжності чисельного розв'язку МСЕ до точного на решітках тетраедрально-октаедральної структури при розв'язанні граничних задач для диференціальних рівнянь еліптичного типу. Для програмної алгоритмізації MCE у роботах [1-4] отримано формули чисельного інтегрування по області лінійного октаедра, які дозволяють знаходити точно елементи локальної матриці жорсткості лінійного октаедра з кусково-лінійними [1-3], квадратичними [4] та тригонометричними базисними функціями [5]. У роботі [6] отримано кубатурну формулу для квадратичного октаедра 3 поліноміальним четвертого порядку базисом [7], яка дозволяе знаходити точно елементи локальної матриці жорсткості квадратичного октаедра.

Виділення не вирішених раніше частин загальної проблеми. Для чисельної алгоритмізації МCE у просторових областях, які дискретизовано решіткою тетраедрально-октаедральної структури, необхідно отримати кубатурні формули на лінійному та квадратичному октаедрах для обчислення відповідних елементів локальних матриць мас. Додатково існуе можливість побудувати загальну формулу чисельного інтегрування по області октаедра, яка буде точною для тривимірних алгебраїчних поліномів до степеня $\mathrm{n}$ включно, оптимальною за кількістю вузлів інтерполяції та часовою складністю процесу обчислень.

Тоді формула (1) запишеться у вигляді:

$$
\iiint_{\Omega} f(x, y, z) d x d y d z \approx \sum_{s=1}^{6} A_{s} f\left(a_{s}\right)+\sum_{s=1}^{12} B_{s} f\left(b_{s}\right)+\sum_{s=1}^{8} C_{s} f\left(c_{s}\right)+D_{0} f\left(d_{0}\right),
$$

де $A_{s}, B_{s}, C_{s}, D_{0}$ - вагові коефіціенти.

Будемо говорити, що дана кубатурна формула має алгебраїчний порядок точності $n$, якщо вона $\epsilon$ точною для всіх многочленів степеня не вище $n$ та не $є$ точною хоча $б$ для одного 3 многочленів степеня $n+1$.

Похибкою кубатурної формули (1) будемо вважати величину

$$
\max _{f \in C^{n}(\Omega)}\left|R_{n+1}(f)\right| \text {, де } R_{n+1}(f)=\iiint_{\Omega} f(X) d X-I_{R}(f),
$$

- залишковий член формули (1), $d X=d x d y d z$ - елемент об'єму.

Якщо $f(X)=f(x, y, z)$, то за формулою Тейлора для $f(X)$ в околі точки $X_{0} \in \Omega$ із залишковим членом у формі Лагранжа маємо:

$$
f(X)=\sum_{s=1}^{n} \sum_{|\beta|=s} \frac{1}{\beta !} \frac{\partial^{|\beta|} f\left(X_{0}\right)}{\partial X^{\beta}}\left(X-X_{0}\right)^{\beta}+\sum_{|\beta|=n+1} \frac{1}{\beta !} \frac{\partial^{|\beta|} f\left(X_{0}+\theta\left(X-X_{0}\right)\right)}{\partial X^{\beta}}\left(X-X_{0}\right)^{\beta},
$$

де $\beta=\beta(i, j, k)$ - мультиіндекс, $|\beta|=i+j+k, i, j, k=\overline{1,3}, \beta !=i ! j ! k !, 0<\theta<1-$ деяке число.

Тоді

$$
R_{n+1}(f)=\iiint_{\Omega} \sum_{\mid \beta=n+1} \frac{1}{\beta !} \frac{\partial^{|\beta|} f\left(X_{0}+\theta\left(X-X_{0}\right)\right)}{\partial X^{\beta}}\left(X-X_{0}\right)^{\beta} d X .
$$

Оцінимо рівність (3), враховуючи, що $\left|X-X_{0}\right| \leq 2$ для довільних точок $X, X_{0} \in \Omega$ :

$$
\left|R_{n+1}(f)\right| \leq \iiint_{\Omega} \sum_{|\beta|=n+1}\left|\frac{1}{\beta !} \frac{\partial^{|\beta|} f\left(X_{0}+\theta\left(X-X_{0}\right)\right)}{\partial X^{\beta}}\left(X-X_{0}\right)^{\beta}\right| d X \leq \leq \iiint_{\Omega} \sum_{|\beta|=n+1}\left|\frac{2^{\beta}}{\beta !} \frac{\partial^{|\beta|} f\left(X_{0}+\theta\left(X-X_{0}\right)\right)}{\partial X^{\beta}}\right| d X=K,
$$

де $K=$ const $>0$.

Тобто для кубатурної формули порядку $n$ всі величини $R_{i}(f)=0$ при $i \leq n$, а $R_{n+1}(f)>0$.

Для поліномів $P_{n}(x, y, z)=\sum_{|\alpha|=0}^{n} a_{i j k} x^{i} y^{j} z^{k} \quad(n \leq 7)$, де $a_{i j k}$ - коефіціенти, $\alpha=\alpha(i, j, k)-$ мультиіндекс, $|\alpha|=i+j+k, i, j, k=\overline{1,3}$, формула (2) $\mathrm{e}$ точною, якщо 


$$
\begin{aligned}
\iiint_{\Omega} P_{n}(x, y, z) d x d y d z & =\frac{4}{3} a_{000}+\frac{2}{15}\left(a_{200}+a_{020}+a_{002}\right)+\frac{4}{105}\left(a_{400}+a_{040}+a_{004}\right)+\frac{2}{315}\left(a_{220}+a_{022}+a_{202}\right)+ \\
& +\frac{1}{63}\left(a_{600}+a_{060}+a_{006}\right)+\frac{1}{945}\left(a_{420}+a_{402}+a_{240}+a_{042}+a_{204}+a_{024}\right)+\frac{1}{5670} a_{222} .
\end{aligned}
$$

У роботі [6] показано, що рівність (4) приводить до системи рівнянь:

$$
\left\{\begin{array}{l}
6 A+12 B+8 C+D=\frac{4}{3} \\
A p^{2}+4 B q^{2}+4 C r^{2}=\frac{1}{15} \\
A p^{4}+4 B q^{4}+4 C r^{4}=\frac{2}{105} \\
2 B q^{4}+4 C r^{4}=\frac{1}{105} ; \\
2 A p^{6}+8 B q^{6}+8 C r^{6}=\frac{1}{21} \\
4 B q^{6}+8 C r^{6}=\frac{1}{315} \\
C r^{6}=\frac{1}{45360} ;
\end{array}\right.
$$

в якій рівновіддалені від центра октаедра вузли мають рівні вагові коефіцієнти, тобто $A_{1}=\ldots=A_{6}=A$, $B_{1}=\ldots=B_{12}=B, C_{1}=\ldots=C_{8}=C$.

Отже, відповідна кубатурна фрормула має вигляд:

$$
\iiint_{\Omega} f(x, y, z) d x d y d z \approx A \sum_{s=1}^{6} f\left(a_{s}\right)+B \sum_{s=1}^{12} f\left(b_{s}\right)+C \sum_{s=1}^{8} f\left(c_{s}\right)+D \cdot f\left(d_{0}\right)
$$

Нижче наведено параметри всіх отриманих квадратур, які задовольняють умові, коли вагові коефіцієнти приймають додатні значення.

Кубатура $n=3, N=6, A=\frac{2}{9}, B=C=D=0, p=\sqrt{0.3}$ [4].

Відмітимо, що дана кубатура є мінімальною за кількістю вузлів, яка є точною для поліномів третього ступеня. Усі вузли $a_{s}$ належать області октаедра.

Кубатура $n=5, N=14$ має два розв'язки, які отримано з (5) при $B=D=0$. А саме:

1) $p_{1}=\frac{1}{231} \sqrt{24255-231 \sqrt{1785}}, r_{1}=\frac{1}{273} \sqrt{17199+273 \sqrt{1785}}$,

$$
A=\frac{61}{480}+\frac{1}{480} \sqrt{1785} ; C=\frac{137}{1920}-\frac{1}{640} \sqrt{1785} ;
$$

2) $p_{2}=\frac{1}{231} \sqrt{24255+231 \sqrt{1785}}, r_{2}=\frac{1}{273} \sqrt{17199-273 \sqrt{1785}}$,

$$
A=\frac{61}{480}-\frac{1}{480} \sqrt{1785} ; C=\frac{137}{1920}-\frac{1}{640} \sqrt{1785} .
$$

Зауважимо, що у випадку, коли вузлам $a_{s}, c_{s}$ відповідають значення $p_{1}, r_{1}$, вузли $c_{s}$ не належать області октаедра. Орієнтовна кількість вузлів інтерполяції $N \cong\left[\frac{(k+n) !}{(k+1) ! n !}\right]$, де $n-$ ступінь полінома, $k-$ розмірність простору [8], при $n=5, k=3$ дорівнюе 10. Спроби зменшити кількість вузлів інтерполяції в формулі (6) приводять до несумісної системи рівнянь (5).

Кубатура $n=7, N=27$ має два розв'язки [6]:

1) $p_{1}=\sqrt{\frac{948+\sqrt{2370}}{1830}}, q_{1}=\sqrt{\frac{168-\sqrt{2370}}{834}}, r_{1}=\sqrt{\frac{276+5 \sqrt{2370}}{546}}$,

$A=\frac{4550}{89373}-\frac{142325}{889618842} \sqrt{2370} ; B=\frac{3926}{89373}+\frac{14507}{22521996} \sqrt{2370}$;

$C=\frac{324461}{6256110}-\frac{47963}{45043992} \sqrt{2370} ; D=\frac{89492}{1042685}+\frac{777893}{444809421} \sqrt{2370}$;

2) $p_{2}=\sqrt{\frac{948-\sqrt{2370}}{1830}}, q_{2}=\sqrt{\frac{168+\sqrt{2370}}{834}}, r_{2}=\sqrt{\frac{276-5 \sqrt{2370}}{546}}$,

$A=\frac{4550}{89373}+\frac{142325}{889618842} \sqrt{2370} ; B=\frac{3926}{89373}-\frac{14507}{22521996} \sqrt{2370} ;$

$C=\frac{324461}{6256110}+\frac{47963}{45043992} \sqrt{2370} ; D=\frac{89492}{1042685}-\frac{777893}{444809421} \sqrt{2370}$. 
При цьому коли вузлам $a_{s}, b_{s}, c_{s}$ відповідають значення $p_{1}, q_{1}, r_{1}$, вузли $c_{s} \notin \Omega$. $\mathrm{Y}$ випадку, коли вузлам $a_{s}, b_{s}, c_{s}$ відповідають значення $p_{2}, q_{2}, r_{2}$, вузли $b_{s} \notin \Omega$.

Орієнтовна кількість вузлів інтерполяції $N \cong 21$ при $n=7, k=3$. Спроби зменшити кількість вузлів інтерполящії в фрормулі (6) приводять до несумісної системи рівнянь (5).

Висновки і пропозиції. У роботі побудовано кубатурну формулу по області октаедра, точність якої можна збільшити до сьомого алгебраїчного порядку додаванням певної групи вузлів інтерполяції, та визначено оптимальні параметри формули чисельного інтегрування. Отримана

формула задовольняе умові додатних вагових коефіцієнтів, а також є мінімальною за кількістю вузлів інтерполяції при $n=3$ та має два різних набори координат вузлів та вагових коедріціентів при $n=\{5,7\}$. При $n=5$ існуе можливість обрати формулу, вузли якої належать області інтегрування та відповідають значенням параметрів $p_{2}, r_{2}$. При $n=7$ незалежно від вибору параметрів кубатурної фороули одна 3 груп вузлів не належить області інтегрування. Побудована кубатурна формула може бути застосована при розв'язанні граничних задач математичної фрізики для об'ємних областей, які дискретизовані решіткою тетраедрально-октаедральної структури.

\section{Список літератури:}

1. Grosso R., Greiner G. Hierarchical Meshes for Volume Data. Computer Graphics International 1998: Proceeding of the Conference (Washington, July 22-27, 1998). Washington, 1998. P. 761-771.

2. Greiner G., Grosso R. Hierarchical tetrahedral-octahedral subdivision for volume visualization. The Visual Computer. Berlin, 2000. Vol. 16. I. 6. P. 357-369.

3. Мотайло А.П. Геометричне моделювання скалярних та векторних полів на решітках тетраедрально-октаедральної структури : дис. ... канд. техн. наук : 18.10.19. Дніпро, 2019. 164 с.

4. Мотайло А.П., Білоусова Т.П. Побудова кубатурної формули для октаедра. Сучасні енергетичні установки на транспорті, технології та обладнання для їх обслуговування : матеріали 10-ї міжнародної науковопрактичної кондеренції (Херсон, 12-13 вересня 2019 р.). Херсон, 2019. С. 277-280.

5. Мотайло А.П., Алексенко В.Л. Кубатурна фрормула по октаедру для тригонометричного полінома окремого виду. Перспективні напрялки сучасної електроніки, інбормаційних $i$ колпютерних систел : матеріали IV-ї всеукраїнської науково-практичної конференції (Дніпро, 27-29 листопада 2019 р.). Дніпро : ДНУ, 2019.C. 58-60.

6. Мотайло А.П. Кубатурна формула для октаедра сьомого алгебраїчного порядку точності. Прикладні питання математичного моделювання. Т. 3. № 2. Ч. 2. Херсон, 2020. С. 184-194.

7. Мотайло А.П. Побудова гармонічного базису квадратичного октаедра. Сучасні технологї пролислового комплексу : матеріали V Міжнародної науково-практичної конференції (Херсон, 10-15 вересня 2019 р.). Херсон : ХНТУ, 2019. С.178-180.

8. Мысовских И.П. О построении кубатурных формул для простейших областей. Журнал вычислительной математики и мателатической фбизики. 1964. Т. 4. № 1. С. 3-14.

\section{References:}

1. Grosso, R., \& Greiner, G. (1998) Hierarchical Meshes for Volume Data. Computer Graphics International: International Conference (Germany, Hannover, June 22-24, 1998). Washington: IEEE Computer Society Press, pp. 761-771.

2. Greiner G., Grosso R. (2000) Hierarchical tetrahedral-octahedral subdivision for volume visualization. The Visual Computer. Berlin, vol. 16, i. 6, pp. 357-369.

3. Motailo A.P. (2019) Heometrychne modeliuvannia skaliarnykh ta vektornykh poliv na reshitkakh tetraedralnooktaedralnoi struktury: dys. ... kand. tekhn. nauk: 18.10.19. Dnipro, 164 p.

4. Motailo, A. P., \& Bilousova T. P. (2019) Pobudova kubaturnoi formuly dlia oktaedra. Proceedings of the Suchasni enerhetychni ustanovky na transporti, tekhnolohii ta obladnannia dlia yikh obsluhovuvannia: 10-ta mizhnarodna naukovo-praktychna konferentsiia (Kherson, September 12-13, 2019). Kherson: KDMA, pp. 277-280.

5. Motailo, A. P., \& Aleksenko V. L. (2019) Kubaturna formula po oktaedru dlia tryhonometrychnoho polinoma okremoho vydu. Proceedings of the Perspektyvni napriamky suchasnoielektroniky, informatsiinykh ikompiuternykh system: IV vseukrainska naukovo-praktychna konferentsiia (Dnipro, November 27-29, 2019). Dnipro: DNU, pp. 58-60.

6. Motailo A.P. (2020) Kubaturna formula dlia oktaedra somoho alhebraichnoho poriadku tochnosti. Prykladni pytannia matematychnoho modeliuvannia, t. 3, no. 2, ch. 2. Kherson, pp. 184-194.

7. Motailo A.P. (2019) Pobudova harmonichnoho bazysu kvadratychnoho oktaedra. Proceedings of the Suchasni tekhnolohii promyslovoho kompleksu: V Mizhnarodna naukovo-praktychna konferentsiia (Kherson, September 10-15, 2019). Kherson: KNTU, pp. 178-180.

8. Myisovskih I.P. (1964) O postroenii kubaturnyih formul dlya prosteyshih oblastey. Zhurnal vyichislitelnoy matematiki i matematicheskoy fiziki, t. 4, no. 1, pp. 3-14. 